\title{
Efficient Walking Gait Generation via Principal Component Representation of Optimal Trajectories: Application to a Planar Biped Robot with Elastic Joints.
}

\author{
Gian Maria Gasparri ${ }^{1}$, Silvia Manara ${ }^{2}$, Danilo Caporale ${ }^{1}$, Giuseppe Averta ${ }^{1}$, Manuel Bonilla ${ }^{1}$, \\ Hamal Marino $^{1}$, Manuel Catalano ${ }^{3}$, Giorgio Grioli ${ }^{3}$, Matteo Bianchi ${ }^{1}$, Antonio Bicchi ${ }^{3}$ and Manolo Garabini ${ }^{1}$
}

\begin{abstract}
Recently, the method of choice to exploit robot dynamics for efficient walking is numerical optimization (NO). The main drawback in NO is the computational complexity, which strongly affects the time demand of the solution. Several strategies can be used to make the optimization more treatable and to efficiently describe the solution set. In this work, we present an algorithm to encode effective walking references, generated off-line via numerical optimization, extracting a limited number of principal components and using them as a basis of optimal motions. By combining these components, a good approximation of the optimal gaits can be generated at run time. The advantages of the presented approach are discussed, and an extensive experimental validation is carried out on a planar legged robot with elastic joints. The biped thus controlled is able to start and stop walking on a treadmill, and to control its speed dynamically as the treadmill speed changes.
\end{abstract}

\section{INTRODUCTION}

$\mathbf{T}$ HE energy efficiency of robotic locomotion is strongly related to the careful exploitation of the natural dynamics of the robots. For example, looking at passive and heavily under-actuated bipeds, a fine tuned design and control is crucial to get [1], [2], [3] highly efficient systems. However, these robots still exhibit limited flexibility if compared to fully actuated humanoids, which, on the other hand, present a high Cost of Transport (CoT) [1], [4]. Main reasons for this poor energy performance of humanoids can be ascribed to the mismatch between the complexity of robot dynamics and the oversimplified modeling (e.g. cart-table, spring loaded inverted pendulum, etc.), which is usually employed for robotic locomotion planning. [5], [6], [7] .

Such a mismatch is even more evident in the emerging generation of elastic joint robots (e.g. [8], [9]) since the actuation

Manuscript received: September, 10, 2017; Revised: December, 22, 2018; Accepted: January, 31, 2018.

This paper was recommended for publication by Editor Allison M. Okamura upon evaluation of the Associate Editor and Reviewers' comments

This work was supported by the European Commission project (FP7 framework) Walk-Man n FP7-ICT-2013-10 under Grant 611832

${ }^{1}$ G. Gasparri , D. Caporale, G. Averta, M. Bonilla, H. Marino, M. Bianchi, M. Garabini are with Centro di Ricerca "E. Piaggio", Università di Pisa, Italy gasparrigianmaria@gmail. com

2 S. Manara is with Dept. of Civil and Industrial Eng., Università di Pisa, Italy,

${ }^{3}$ M. Catalano , G. Grioli and A. Bicchi are with Dept. of Adv. Robotics, Istituto Italiano di Tecnologia, Genoa, Italy

Digital Object Identifier (DOI): see top of this page.

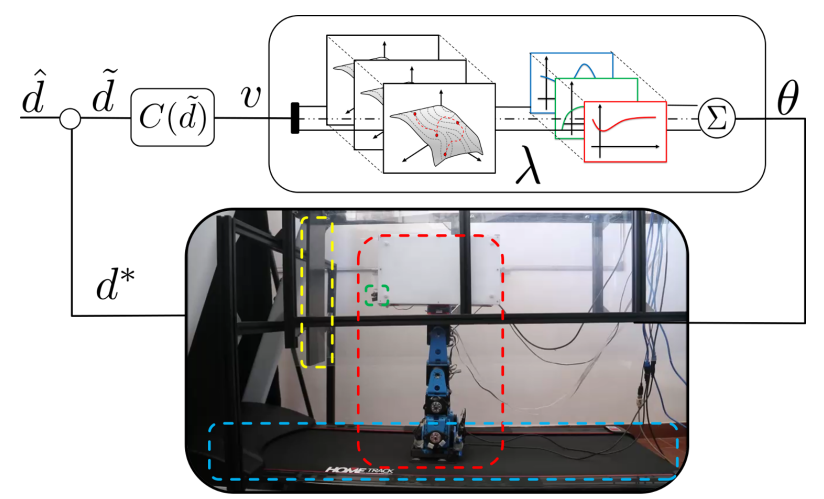

Figure 1. A six DoF biped robot (in red), named SoftLegs, walks on a treadmill (in blue) and a target (in yellow) is placed in front of it. An infrared sensor (in green) retrieves the current distance $d^{*}$ of the robot from the target. Given the desired distance $\hat{d}$, the task goal is to regulate the error $\tilde{d}=\hat{d}-d^{*}$ while the treadmill speed changes from $0.3 \mathrm{~km} / \mathrm{h}$ to $0.7 \mathrm{~km} / \mathrm{h}$. The proposed control approach includes a PI controller $C(\tilde{d})$ that provides as output the forward speed of the robot $v$. Then the principal component combination generates the desired motor trajectories $\theta$ that approximate the optimal (i.e. efficient) gait at the given speed $v$.

compliance, purposefully introduced in the mechanical design to guarantee robustness [10], efficiency [11], peak performance [12], is usually neglected or not properly taken into account.

NO could represent a viable approach to tackle this issue and hence take advantage of the full robot dynamics for motion planning. Recently, in [13] NO was applied to plan the walking motion of a 23 Degree-of-Freedom (DoF) humanoid robot. The results show a cost of transport slightly above 1, which currently represents the best performance achievable with fully actuated humanoids. However, NO comes with a cost, which is related to time demand: e.g. the footstep optimization presented in [13] requires from 910 to 380 seconds. For these reasons, to successfully use NO for online applications, a suitable combination of off-line and on-line computations is required. This usually implies the off-line evaluation of a large set of trajectories and their encoding. For example, in [14] relevant features of optimal walking trajectories (e.g. cost of transport and trajectory of the center of mass) were encoded via quadratic functions of the initial state and footsteps.

In [15] the authors moved from an off-line dataset of optimal gaits, and applied supervised learning to identify state- 
variable feedback control policies to manage speed transition and disturbance rejection.

In [16] walking gaits were synthesized via motion primitives [17]. A similar approach is commonly used also in computer graphics [18], [19]. In [18] Principal Component Analysis (PCA) was employed to encode a large set of behaviors (from an existing motion capture database) in motion primitives to animate a 60 DoF character. In [19] reinforcement learning was adopted to synthesize locomotion controllers that correspond to specific motion skills (e.g. jumping movements extracted from a motion capture database).

In [16] parametric motion primitives were learned from a dataset of optimized trajectories. Once the motion primitives were defined, their parameters were used as optimization variables to synthesize trajectories not belonging to the original dataset.

In this paper, we present an approach that allows to drastically reduce the space complexity of the original data set. Furthermore, it also enables the robot to update its stride online and to synthesize gaits not belonging to the original data set (avoiding further optimizations that are required e.g. in [16]).

More specifically, we propose a technique that leverages upon NO (see e.g. [13]), and dimensionality reduction of PCA for on-line planning of efficient locomotion of robots with elastic actuation. We built a set of optimized gaits for different speeds and swinging foot clearances. The gaits we obtained minimize robot energy consumption fulfilling both stability and dynamic feasibility requirements, while a reduced representation of the set of optimal gaits was identified through PCA. Similarly to [16], the relationship between the principal component (PC) weights and task parameters (namely, forward speed and step clearance) was approximated by a parametric representation, named Component Map (CM), while a mostly feed-forward strategy was used to preserve the mechanical compliance of the system introduced by design as in [20] - which is not generally guaranteed by learning a feedback policy (which was described e.g. in [15]).

One of the main contributions of our work is the extensive numerical and experimental validation of the method on a planar biped robot powered by compliant actuators (see Fig. 1) named SoftLegs. Numerical results show that: (i) only two PCs are needed to satisfactorily reconstruct a walk (explained variance 99\%) and, (ii) CMs allow to reduce the complexity of the original data set by two orders of magnitude.

Finally, experiments demonstrated that with our techniques the robot was able to successfully start and stop walking, perform transitions between different forward speeds, and walk on a treadmill while the velocity of the belt changed (see Fig. $1)$.

\section{Problem Definition}

In this section we compare the CoT given by optimized walking gaits with the CoT obtained by using a classical ZMP based method. The comparison is carried out on a six DoF planar biped (a scheme is reported in Fig. 2(a),2(b))

The biped is composed of two planar three-DoF legs, connected to a pelvis on which a one-DoF trunk is mounted.

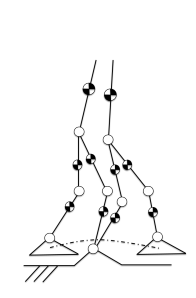

(a)

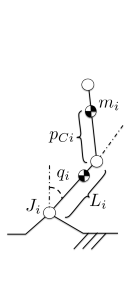

)

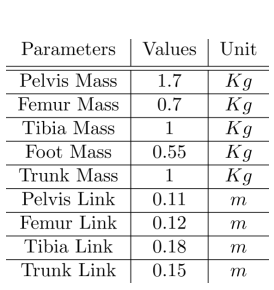

(b)

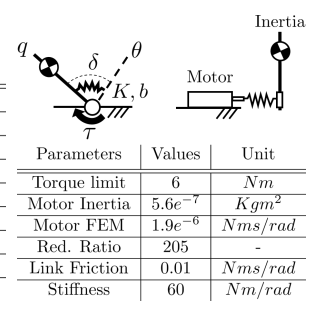

(c)
Figure 2. Model of the biped: biped kinematics 2(a), kinematic and dynamic parameters 2(b), scheme and parameters of the SEA 2(c). The link and the motor displacement are represented by $q$ and $\theta$ respectively, $k$ is the elastic transmission stiffness and $b$ the motor damping (more details in the appendix). Quantities in table are named in analogy with their biological counterparts.

Each joint of the robot is powered by a Series Elastic Actuator (SEA) [21]. The parameters of the motor dynamics are showed in Fig.2(c) (details in appendix).

Given desired forward speed $v$ and swing foot clearance $f_{h}$, we derive, in accordance to [22], the robot step length:

$$
s=\alpha \nu^{\beta},
$$

where $s=S / l$ is the normalized step length, $l$ is the leg length and $\nu=v / \sqrt{l g}$ is the normalized speed. The stride parameter sets used in the study are: $f_{h}=[0.01,0.03] \mathrm{m}$ with a granularity of $5 \mathrm{~mm}$, and $v=[0.04,0.5] \mathrm{m} / \mathrm{s}$ with a granularity of $1 \mathrm{~cm} / \mathrm{s}$. These choices resulted in 485 trajectories. The upper bound of the velocity range has been chosen to include the walk-to-run transition speed ${ }^{1}$ in order to guarantee to span dynamic gaits, while the lower bound was selected to allow a smooth transition to the standing posture. The swingfoot clearance range has been chosen to avoid compass gaits (discovered by the optimizer for $f_{h}=0$ ) by preferring more practical gaits up to a step height equal to ten percent of the leg length.

For the sake of clarity, a detailed formulation of the optimal control problem to derive a walking gait is provided in appendix. As examples we show in Fig.3 the photo sequence of two optimal walks.
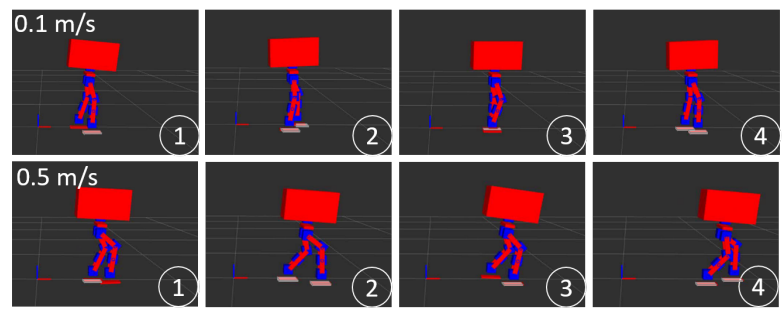

Figure 3. Photo sequence of two optimal gaits for two different forward speeds: on the top $v=0.1 \mathrm{~m} / \mathrm{s}$ and on the bottom $v=0.5 \mathrm{~m} / \mathrm{s}$. The feet are colored in white when in contact with the ground.

The ZMP-based trajectories were determined according to [5]. Combining step parameters and centre-of-mass evolution, it is possible to obtain the link trajectories, and, through inverse

\footnotetext{
${ }^{1}$ The walk-run transition speed, defined as the speed at which running becomes more convenient than walking [23], has been determined with another optimization campaign whose results is not included due to space limits
} 
dynamics, motor positions and torques to finally evaluate the CoT.

Fig. 4(a) presents the values of the CoT of the two approaches over a range of forward speeds. The simulations show that the NO gaits perform at least four times better if compared to the ZMP based gaits. Fig.4(c) shows the joint torques during the walking cycle for the case with $\nu=0.1 \mathrm{~m} / \mathrm{s}$ and $f_{h}=0.03 \mathrm{~cm}$. It is possible to notice that there are minor

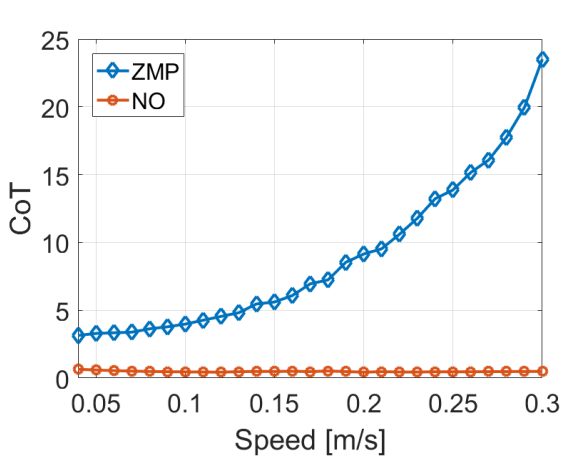

(a)

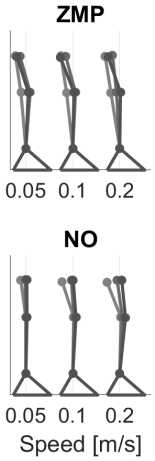

(b)
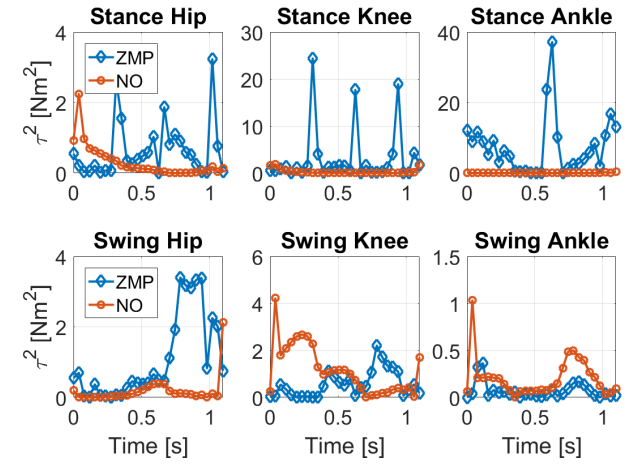

(c)

Figure 4. 4(a) Comparison between ZMP-based and NO-based CoT at different speed averaged over the swing/foot step height. 4(b) Stance leg configuration at different speed. 4(c) Squared torque evolution of Stance (top) and Swing (bottom) leg for a ZMP-based and NO-based step for two different forward speed $v=0.1 \mathrm{~m} / \mathrm{s}$ and $f_{h}=0.03 \mathrm{~m}$.

differences in the swinging leg squared torques of the two methods. Conversely the stance leg squared torques of $\mathrm{NO}$ are substantially lower than ZMP ones. This because NO fully exploits the leg singularity in the central part of the motion (see Fig.4(b)). The application of NO presents one major limitation: for the majority of cases of practical interest the optimization problems are too complex to be solved at run time.

\section{OPTIMAL GAIT ENCODING VIA PRINCIPAL \\ COMPONENTS}

In this section we apply PCA to encode the data set of optimal trajectories into a small set of PCs and their weights. Then we interpolate the dependence of the weights on the walk parameters through a CM.

The starting dataset is determined as follows. Assume that: (i) the robot is composed of $n_{j}$ joints, (ii) the trajectories are described by $n_{t}$ sample times, (iii) the walking task is described by $n_{p}$ parameters (e.g. forward speed, step height, lateral speed, etc...) and (iv) $n_{s}(i)$ parameter samples are needed to explore the range of the $i$-th parameter. Hence, the starting data set can be stored in a matrix $\Sigma \in \mathbb{R}^{m_{j t} \times m_{s p}}$, where $m_{j t}=n_{j} \times n_{t}$ and $m_{s p}=\Pi_{i=1}^{n_{p}} n_{s}(i) . \Sigma$ has been evaluated accordingly to Sec. II $\left(n_{p}=2, n_{s}=\{5,95\}\right)$. In Fig. 5 we report the complete set of trajectories for Hip, Knee, and Ankle used in this study. Fig.5 suggests a
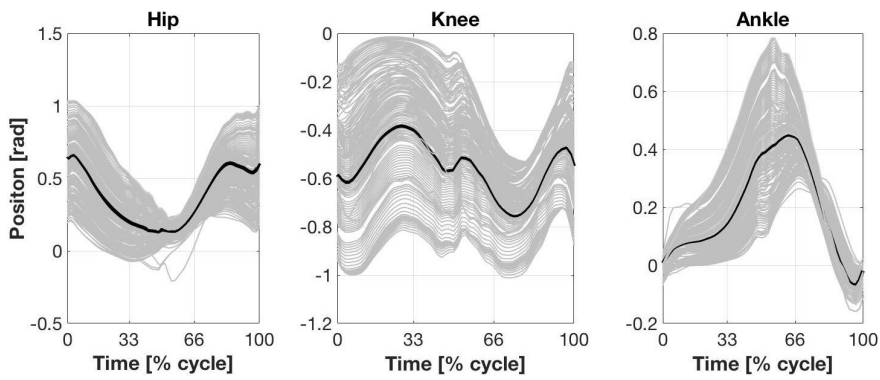

Figure 5. Motor trajectories of $\Sigma$ (in gray) and a representative trajectory (in black).

strong correlation between motor trajectories, motivating the application of motion primitives.

\section{A. Principal Components Analysis}

Among the many methods proposed to identify motion primitives (e.g. radial basis function, gaussian process, etc), we decide to rely on PCA which represents an effective compromise between complexity and computational cost. PCA uses an orthogonal transformation to convert a set of possibly correlated variables, to which the mean value of each observation is subtracted, into a set of values of linearly uncorrelated components, called PCs. In order to apply the PCA to the robot trajectories we proceed as follows. Each motor trajectory $\theta_{j} \in \mathbf{R}^{n_{t}}$ of the starting set is split into two components: its mean value $\bar{\theta}_{j} \in \mathbf{R}$ and a null mean vector $\tilde{\theta}_{j}=\theta_{j}-\bar{\theta}_{j}$ for $j \in\left\{1, \ldots, n_{j}\right\}$. Hence $\Sigma$ can be described by two matrices: $\Sigma_{m} \in \mathbf{R}^{m_{j t} \times m_{s p}}$ and $\Sigma_{p} \in \mathbf{R}^{n_{j} \times m_{s p}}$. Each column of $\Sigma_{m}$ is a vector composed of $\left[\tilde{\theta}_{1}, \ldots, \tilde{\theta}_{n_{j}}\right]^{T}$. Each column of $\Sigma_{p}$ is a vector composed of $\left[\bar{\theta}_{1}, \ldots, \bar{\theta}_{n_{j}}\right]^{T}$ that represents the robot mean pose (hereinafter Pose) of the corresponding movement (column) in $\Sigma$. We applied PCA to $\Sigma_{m}$ and $\Sigma_{p}$ for the case study of the planar biped robot with elastic joints $\left(n_{j}=6\right.$, $\left.n_{t}=30\right)$. The variance explained by the PCs, reported in

Table I

EXPLAINED VARIANCE AS A FUNCTION OF THE COMBINATION OF AN INCREASING NUMBER OF PRINCIPAL COMPONENTS.

\begin{tabular}{|c|c|c|c|}
\hline N PC & $\Sigma_{m}$ & $\Sigma_{p}$ & RMSE \\
\hline \hline 1 & $87.04 \%$ & $98.81 \%$ & 0.06 \\
\hline 2 & $98.75 \%$ & $\simeq 100 \%$ & 0.017 \\
\hline 3 & $99.43 \%$ & $\simeq 100 \%$ & 0.005 \\
\hline
\end{tabular}

Tab.I, shows that few principal components account for a large amount of variation in both $\Sigma_{m}$ and $\Sigma_{p}$. This leads to hypothesize that all the trajectories of $\Sigma$ can be reconstructed with high precision using only two PCs from a set of 180 PCs.

In the following we evaluate how much the space complexity of $\Sigma$ can be reduced via the application of PCs. Let $O\left(m_{j t} m_{s p}\right)$ and $O\left(n_{j} m_{s p}\right)$ be the space complexities of $\Sigma_{m}$ 
and $\Sigma_{p}$ respectively. Let $p_{k_{m}}$ be the chosen number of PCs to represent the robot movements, $\Sigma_{m}$ can be encoded in the eigenvector matrix $\Sigma_{m, e} \in \mathbb{R}^{m_{j t} \times p_{k_{m}}}$ and in the weight matrix $\Sigma_{m, w} \in \mathbb{R}^{p_{k_{m}} \times m_{s p}}$. Let $p_{k_{p}}$ be the chosen number of PCs to represent the robot Poses, the same procedure can be adopted for $\Sigma_{p}$. This results in a total complexity of $O\left(p_{k_{m}}\left(m_{j t}+m_{s p}\right)+p_{k_{p}}\left(n_{j}+m_{p s}\right)\right)$. Tab.II reports the data related to the case study presented in this paper.

\section{B. Component Maps}

Since in practical cases $m_{s p} \gg m_{j t}$ it is worth to further encode the weight dependence on the task parameters. This can be achieved by exploiting second order polynomial functions. In Fig.6 we present the results of the approximation of the weight dependence on $v$ and $f_{h}$ for both trajectories and Pose.

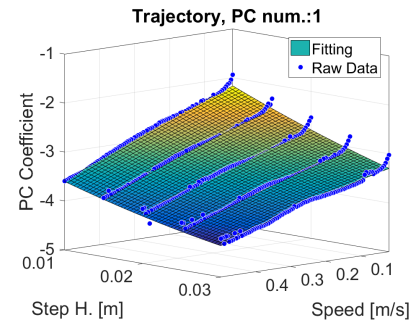

(a) - RMSE 0.038

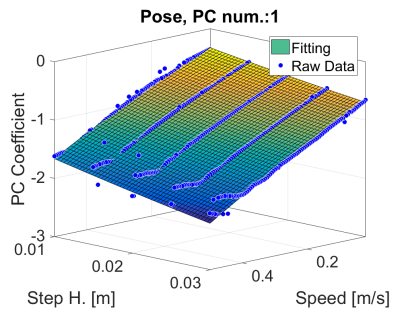

(c) - RMSE 0.043

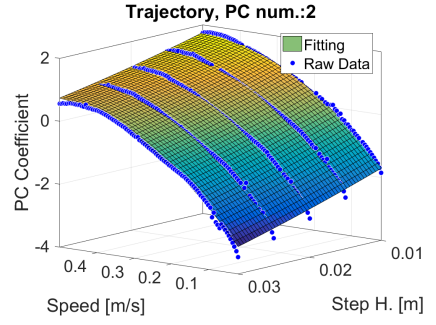

(b) - RMSE 0.023

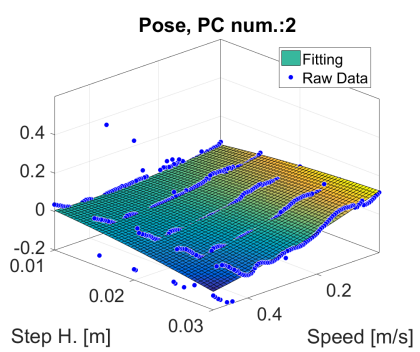

(d) - RMSE 0.035
Figure 6. Principal Component Mapping functions. Surfaces represent the CMs, while the points represents the actual PC weights (Raw Data). First PC weight of $\Sigma_{m, w}$ (a), second PC weight of $\Sigma_{m, w}$ (b), first PC weight of $\Sigma_{p, w}$ (c), second PC weight of $\Sigma_{p, w}$ (d). Each CM is a second order polynomial on each stride variable. The CMs approximate worse both the pose and trajectory PCs for higher speed levels. This explains the RMSE trend in Fig. 7(a). However, this phenomenon occurs just at the boundaries of the explored region of parameters and produces a low RMSE on the whole set.

The very low values of RMSE suggest that the CMs can approximate $\Sigma_{m, w}$ and $\Sigma_{p, w}$. To quantify the level of approximation introduced by applying the CMs we evaluated the RMSE in the reconstruction of the trajectories of $\Sigma$. The results of PCs only and the combination of PCs and CMs are compared in Fig.7(a). Moreover, in Fig.7(b), we report the performance of the two approaches in the reconstruction of a novel set of optimal trajectories not included in $\Sigma$. Both the validation tests show that the reconstruction errors in case of combination of PCs and CMs are comparable to the ones in case of PCs only.

The reduction in the space complexity of $\Sigma_{m, w}$ and $\Sigma_{p, w}$ allowed by the encoding via CMs is evaluated in the following. Assuming that CMs are polynomial functions of order $d$ in

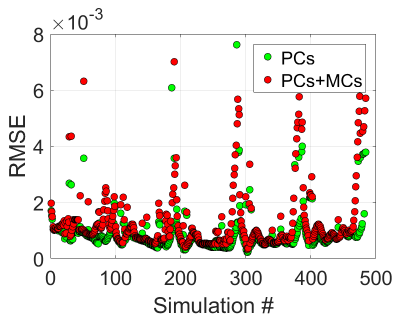

(a)

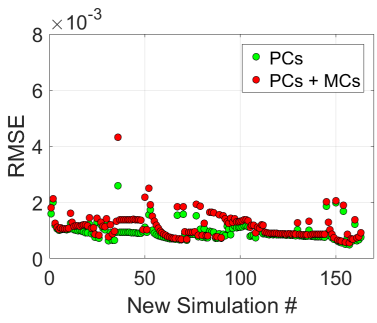

(b)
Figure 7. RMSE of the reconstruction of the optimal trajectories via two PCs (green) and via the combination of PCs and CMs (red). Panel (a) shows the error obtained approximating $\Sigma$, while panel (b) shows the same for a new validation set.

Table II

SPACE COMPleXity COMPARISON

\begin{tabular}{|c|ccc|}
\hline & $\Sigma$ & PCs & PCs + CMs \\
\hline \hline$O(N)$ & $O\left(m_{j t} m_{p s}\right)$ & $\begin{array}{c}O\left(p_{k_{m}}\left(m_{j t}+m_{p s}\right)\right. \\
\left.+p_{k_{p}}\left(n_{j}+m_{p s}\right)\right)\end{array}$ & $\begin{array}{c}O\left(p_{k_{m}}\left(m_{j t}+d^{n_{p}}\right)\right. \\
\left.+p_{k_{p}}\left(n_{j}+d^{n_{p}}\right)\right)\end{array}$ \\
\hline$N$ & 87300 & 2312 & 408 \\
\hline
\end{tabular}

each parameter, an upper bound of the space complexity of each CM is $O\left(d^{n_{p}}\right)$. Hence the total complexity of $\Sigma_{m, w}$ after the encoding becomes $O\left(p_{k_{m}} m_{j t}+p_{k_{m}} d^{n_{p}}\right)$. The same holds for $\Sigma_{p, w}$. The complete complexity analysis is reported in Tab.II.

In conclusion, both PCs and PCs plus CMs show a low RMSE when reconstructing the trajectories of $\Sigma$. Moreover, the space complexity of $\Sigma$ is reduced by one order of magnitude via PCs and two orders of magnitude via PCs plus CMs.

\section{EXPERIMENTAL VALIDATION}

The proposed approach has been experimentally validated in the following cases: (i) constant speed, (ii) variable speed with off-line computation of controls via PC combination, (iii) variable speed with on-line computation of controls via PCs and CMs.

The experimental setup is shown in Fig. 2(a). Each joint of the robot is actuated by a qbmove Advanced (an improved version of [24]). Eight spherical bearings, rolling on two parallel walls, constrain the robot trunk in the sagittal plane. A four bar mechanism drives the ankle joints. Above the centre of rotation of each of them are located the ankle actuators. To retrieve contact forces, four Opto Force sensors ([25]) are mounted in the sole of each foot.

The qbmove actuator dynamics is modelled here as:

$$
I \ddot{\theta}+b \dot{\theta}=-h(\delta, \sigma)+u,
$$

where $\theta, \dot{\theta}, \ddot{\theta}$ are the motor position, speed and acceleration respectively, $\sigma$ is the stiffness adjuster, $I$ is the motor inertia and $b$ is the damping coefficient. $h(\delta, \sigma)$ represents the nonlinear elastic characteristic of the actuator (see [24],[26] for more details). In the following, we consider the linearized actuator stiffness at $\bar{\delta}=0$, i.e.

$$
k=\left.\frac{\partial h(\delta, \sigma)}{\partial \delta}\right|_{\bar{\delta}=0} .
$$



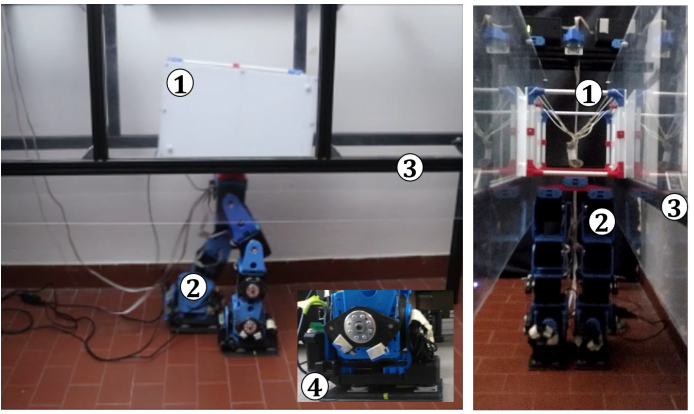

Figure 8. SoftLegs experimental setup: side view (left), and front view (right) The system is composed of two planar legs (2) with three degrees of freedom each and a trunk mounted on the robot pelvis (1). The flat feet are equipped with four force sensors each (4). An external structure (3) constraints the robot to move in its sagittal plane.

Given the stiffness adjuster which reproduces the stiffness used in the optimization, the motor position is set to replicate the optimal behavior returned by the numerical optimization. Reference tracking is guaranteed by a PID control on motor position, i.e. there is no direct feedback of the link position. The motor dynamic parameters are reported in Fig.2(c). The optimization results are concatenated coherently with the robot phase (single or double) to accomplish the locomotion task for several steps. The transition between the initial steady state configuration, and the regime is obtained with few steps generated by low pass filtering the optimal trajectories. The same procedure is used, in reverse, to stop the robot.

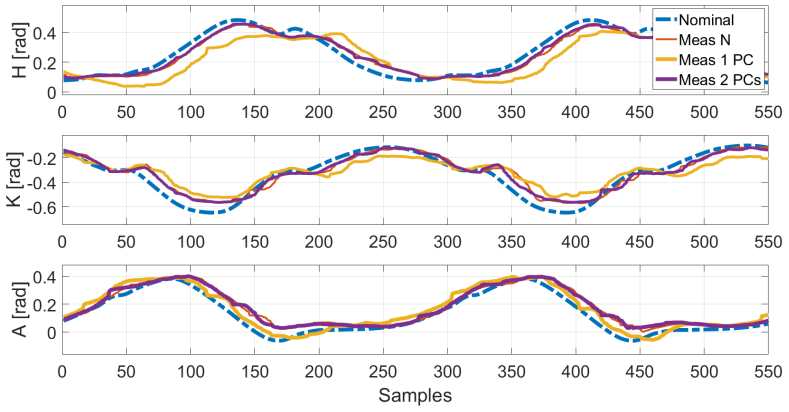

(a)

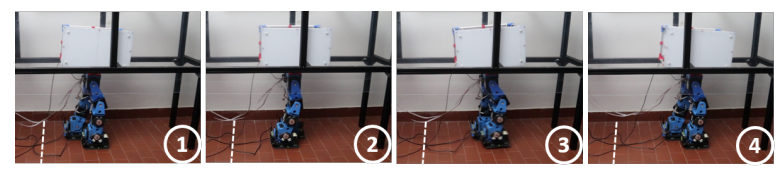

(b)

Figure 9. a) Link measurements of the left leg joint (H,K,A for Hip, Knee and Ankle) obtained by applying: optimal trajectories (Meas N), the first PC (Meas 1 PC) and the combination of the first two PCs (Meas 2 PCs). The signals are compared to the link measurements returned by the optimization (Nominal). b) Photo sequence of the experiment for the speed $v=0.1 \mathrm{~m} / \mathrm{s}$ and foot clearance $f_{h}=0.015 \mathrm{~m}$.

\section{A. Walking with PCs - Constant Speed}

In the first experiment we evaluate the walking capability of the robot driven by (i) the nominal optimal trajectories, (ii) the ones related to the first PC (for both trajectory and pose), and (iii) the ones obtained through the combination of the first and second PCs. The nominal walking conditions are generated for a forward speed of $v=0.1 \mathrm{~m} / \mathrm{s}$ with foot clearance $f_{h}=$ $0.015 \mathrm{~m}$. Fig. 9(a) presents the measured joint values of the left leg. In the case of two PCs the RMSE of the left leg joints per sample, hereinafter JRMSE, is comparable to the one obtained for the nominal case $(\mathrm{JRMSE}=0.0028)$ which is almost the half of the one obtained in the case of application of just the first PC, $(\mathrm{JRMSE}=0.0056)$. Moreover it is possible to argue that the obtained trajectories are dynamically consistent and that a small set of PCs can be successfully applied to reproduce a walk. Fig. 9(b) shows the photo sequence of the experimental result obtained by applying two PCs.

\section{B. Walking with PCs and with PCs and CMs - Speed Variation}

In the second experiment we evaluate the applicability of the PC combination in case of speed variation. Firstly, PCs are used to compute off-line motor references for the cases $v_{1}=$ $0.1 \mathrm{~m} / \mathrm{s}$ and $v_{2}=0.04 \mathrm{~m} / \mathrm{s}$ with $f_{h}=0.015 \mathrm{~m}$. (Other case studies have been tested, e.g. $v_{1}=0.1, v_{2}=0.2 \mathrm{~m} / \mathrm{s}$, but are not reported here due to space limitations). Then, the transition from $v_{1}$ to $v_{2}$ and viceversa is obtained simply switching PC weights and mean poses when the error between the motor references is minimum in norm. The desired and measured link positions are presented in Figs.10(a) and 10(b). These results show that it is possible to modify the robot speed by a proper switch of the references obtained by PC combination.

In the final experiment the biped is placed on a treadmill (Home Track HT2000 min/max speed: $\{0.03,16\} \mathrm{km} / \mathrm{h}$ ) and an on-line control policy adjust the biped speed $v$ in order to maintain a desired position $\hat{d}=0.4 \mathrm{~m}$, while the treadmill velocity is varied (see the attached video). The external structure, which constrains the robot motion, is adapted to the treadmill. An infrared distance sensor, SHARP $2 y 0 a 21$, is mounted on the biped trunk and retrieves the robot distance $d^{*}$ from a target placed in front of the robot (see Fig.1). When the treadmill is activated the robot speed reference $v=C(\tilde{d})$ is provided by a PI controller which uses as input the distance error $\tilde{d}=\hat{d}-d^{*}$. Obtained $v$ and given $f_{h}$ the CMs return the PC weight vector $\lambda$ which is then used to combine the PCs and to obtain the motor references $\theta$. These signals are interpolated and resampled at $T_{s}=0.004 \mathrm{~s}$, i.e. the motor position control runs at $250 \mathrm{~Hz}$ while the speed control loop runs at a sample rate of $100 \mathrm{~Hz}$. Control strategy, PCs and $\mathrm{CMs}$, and signals management are implemented in $\mathrm{C}++/ \mathrm{ROS}$ and run on an Intel Core i7 ThinkPad Notebook. Fig. 1 shows the experimental setup and control scheme while Fig.11 presents the experimental results. In Fig.11(a) $d^{*}$ (Distance in figure) is always within a neighborhood of $\hat{d}$ throughout all the experiment, i.e. the control action successfully adapts the robot gait in response to the varying treadmill speed (mean distance $0.38 \mathrm{~m}$, min distance $0.26 \mathrm{~m}$, $\max$ distance $0.48 \mathrm{~m}$ ). We also notice that $v$ (Speed in figure) is larger than the treadmill speed (T.Speed in figure). This is probably due to the not negligible friction on the supporting structure and to the elasticity of the treadmill belt both not considered in the model.

Two time intervals are colored in red and in green which refer to the slowest and fastest experimental conditions respec- 


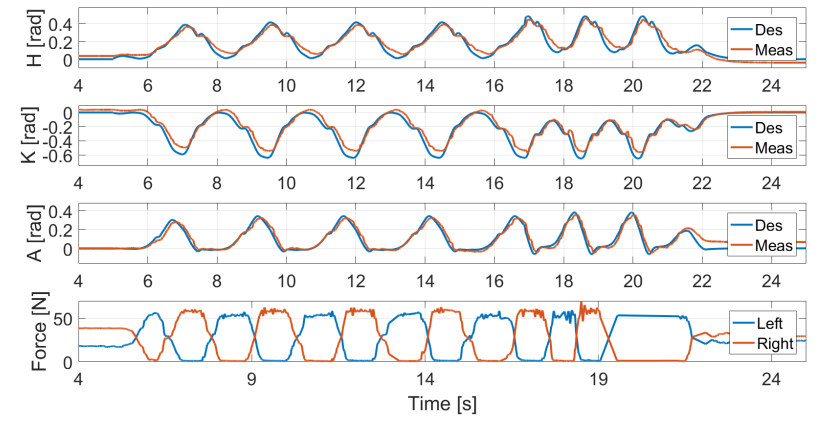

(a)

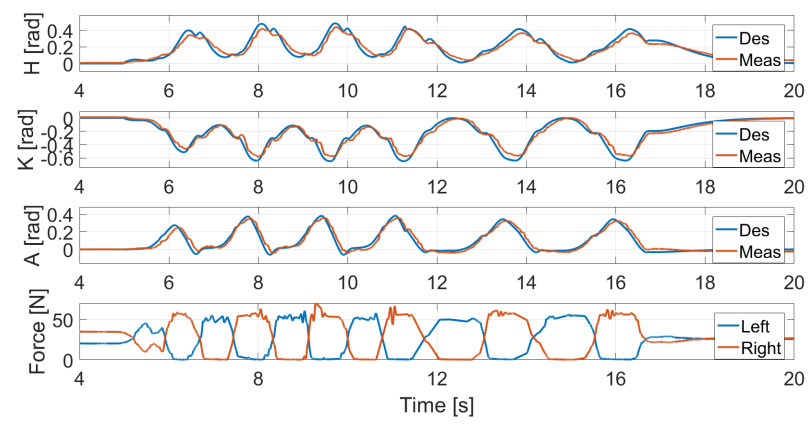

(b)

Figure 10. Experimental robot behaviour during the speed transients: accelerating from $0.04 \mathrm{~m} / \mathrm{s}$ to $0.1 \mathrm{~m} / \mathrm{s}$ (a) and decelerating from $0.1 \mathrm{~m} / \mathrm{s}$ to $0.04 \mathrm{~m} / \mathrm{s}$ (b). Top three plots show the desired (Des) and measured (Meas) values of the Link positions of Hip (H), Knee (K), Ankle (A). Bottom plot shows the contact forces of the two feet.

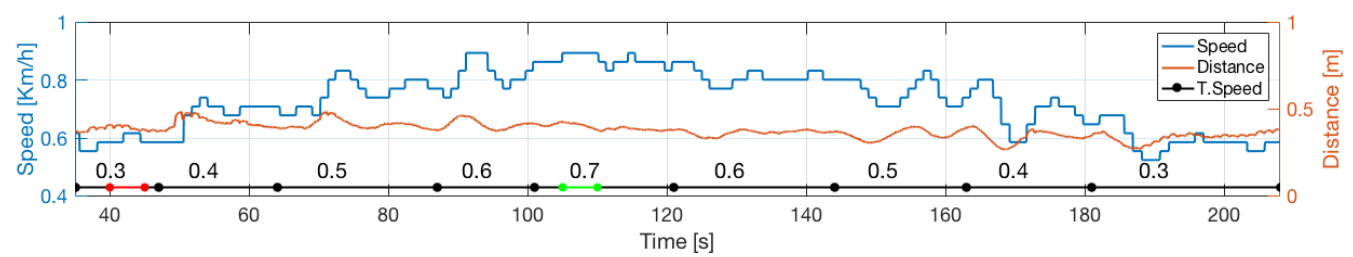

(a)
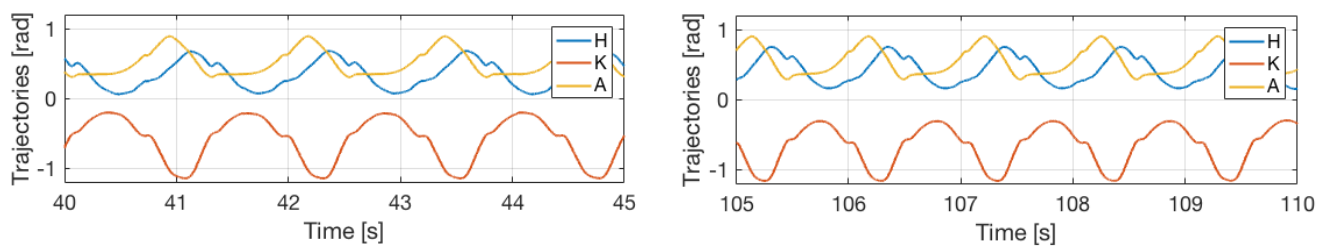

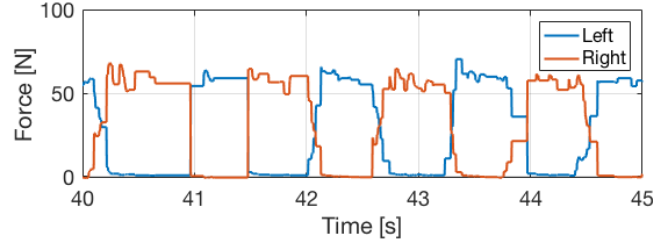

(b)

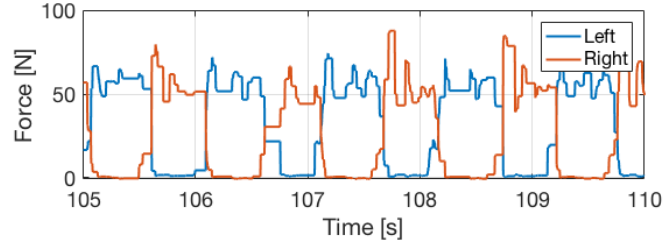

(c)

Figure 11. Experimental results of the treadmill experiments. In (a) the robot Distance (in orange) is showed while the treadmill speed T.Speed is modified. The time line of the varying T.Speed is colored in black. The speed reference provided to the robot, Speed, is reported in blue in figure. On treadmill time line two intervals are colored in red and in green respectively, which refer to the slowest and fastest experimental case, i.e. $0.3 \mathrm{~km} / \mathrm{h}$ in $(\mathrm{b})$ and $0.7 \mathrm{~km} / \mathrm{h}$ in (c). Of these intervals, on the top of the figure the motor positions (H,K,A for Hip, Knee and Ankle) are showed while on the bottom the contact forces (Left and Right foot) are presented.

tively, i.e. $[0.3,0.7] \mathrm{km} / \mathrm{h}$. Fig. 11 (b) shows the desired and current motor position of the left leg joints and contact forces for the slowest speed case. Fig. 11(c) presents analogous signals for the fastest speed case. The experimental result suggests that the considered transition policy and the application of PCs and CMs is indeed admissible. Due to space limitations, in this work we privileged the presentation of experimental validation of the trajectories at different forward speeds over step height since this is the most important parameter toward the distance regulation test.

\section{Robot Performance Evaluation}

By applying PCs plus CMs the robot successfully adapted its stride to match the treadmill speed by using a reduced parameter set and approximating solutions for un- known case studies. It is worth noting that explored tasks cover both static and dynamic gaits. The sum of the norm of the inertial, Coriolis and centrifugal joint torques $\left\|\tau_{M C}\right\|_{2}=[3.52,7.62,12.01,14.37,19.06]^{T} \mathrm{Nm}$, and the sum of the norm of the gravitational joint torques $\left\|\tau_{G}\right\|_{2}=[9.99,12.50,14.74,16.68,18.55]^{T} \mathrm{Nm}$, have been evaluated for the following forward speeds $v=$ $[0.05,0.1,0.15,0.20,0.25] \mathrm{m} / \mathrm{s}$. The results show that at low speeds the gravitational torques play the most important role, while at higher speeds the dynamic torques become larger.

The stability evaluation of the approximated trajectories has not been considered in this paper. A strategy to guarantee the fulfillment of the first-order approximation of the constraints in case of application of the approximated trajectories has been investigated in [27]. Future works will merge this idea with 
the approach presented in this paper.

\section{Conclusions}

In this work numerical optimization has been used to obtain a data set of optimal and efficient locomotion patterns for a six-DoF biped with compliant actuation.

Then PCA and polynomial regression were applied to the trajectories belonging to the data set. This allowed to synthesize gaits that approximate the ones of the data set with a low number of parameters, facilitating the on-line generation of new trajectories.

Finally, this approach has been extensively experimentally validated on a planar biped robot with elastic joints.

Future works will be devoted to make a comparison of our approach with other state-of-art works as [16] and to exploit the reduced representation to optimize trajectories on-line, so as to react to possible external disturbances. The validation of optimal 3D efficient walk for [8] is an ongoing work.

\section{APPENDIX}

Let $n_{a}=6$ be the number of the robot actuated joints, $q \in \mathbb{R}^{n_{a}}$ and $\theta \in \mathbb{R}^{n_{a}}$ be the link and motor position vectors respectively and $\dot{q}, \ddot{q}, \dot{\theta}, \ddot{\theta}$ their time derivatives. The output shaft of each $j$-th motor is connected to the $j$-th joint link via a viscoelastic transmission whose parameters are the stiffness $k_{j}$ and the damping $b_{j}$ with $j \in\left\{1, \ldots, n_{a}\right\}$.

Let $\delta \in \mathbb{R}^{n_{a}}$ be the deflection vector of the elastic elements and $u \in \mathbb{R}^{n_{a}}$ be the motor torques. Defined the stiffness, damping and motor inertia matrices $K=\operatorname{diag}\left(k_{j}\right)$, $B=\operatorname{diag}\left(b_{j}\right), I=\operatorname{diag}\left(I_{j}\right)$ with $I_{j}$ the $j$-th motor inertia, the actuator dynamics is reported in (5).

Let $q_{p} \in \mathbb{R}^{n_{n a}}$ with $n_{n a}=3$ be the vector which uniquely describes the robot position in the sagittal plane, i.e. $q_{p}=\left[p_{x}, p_{y}, \phi\right]$ where $p_{x}$ and $p_{y}$ are the translation and $\phi$ the rotation terms. The dynamics of the system reads:

$$
\left\{\begin{aligned}
M(\xi) \ddot{\xi}+n(\xi, \dot{\xi})+\sum_{i=1}^{n_{p_{i}}} J_{i}^{\top} w_{i} & =Q(k, \delta, \dot{q}) \\
I \ddot{\theta}+B \dot{\theta}+K \delta & =u \\
J_{i} \ddot{\xi} & =\gamma_{i}, i=\left\{1, \ldots, n_{p_{i}}\right\}
\end{aligned}\right.
$$

where $\xi=\left[q_{p}, q\right]^{T}$ is the robot configuration vector and $\dot{\xi}, \ddot{\xi}$ are its time derivatives. $M(\xi)$ is the robot inertia matrix, $n(\xi, \dot{\xi})$ is the vector of nonlinear contributions due to gravity, centrifugal and Coriolis effects and the remaining terms of (4) describe the effects of the exerted forces and motor couplings. In particular the $j$-th component of the vector of generalized forces $Q(K, \delta, \dot{q})$ can be expressed as $Q_{j}=k_{j} \delta_{j}-f_{j} \dot{q}_{j}$ with $f_{j}$ the $j$-th friction term in case the $j$-th DoF is actuated, $Q_{j}=0$ otherwise. $w_{i} \in \mathbb{R}^{3}$ is the planar external wrench acting on the $i$-th support foot at its contact point $p_{i}$, whose Jacobian is $J_{i}$. Finally, $n_{p_{i}}$ is the number of feet that are in contact with the ground.

During the contact, neither sliding nor interpenetration between the foot and the ground are allowed, these result in holonomic constraints on the position of the foot $c_{i}(\xi)$. These constraints must be taken into account in the dynamics, through the additional equations in (6) where $J_{i}=\partial c_{i} / \partial \xi$ and $\gamma_{i}=-\left(\left(\partial J_{i} / \partial \xi\right) \dot{\xi}\right) \dot{\xi}$.

A multiphase formulation is employed to describe the dynamics of the robot through the different contact phases.

The Optimal Control Problem (OCP) that has to be solved in order to obtain the walking trajectories can be stated as follows:

$$
\begin{aligned}
\min _{x(\cdot), u(\cdot), w(\cdot), t_{s}} & \int_{0}^{T_{h}} \frac{\|u(t)\|^{2}}{m g S} d t \\
\text { s.t. } & F_{c}(x(t), \dot{x}(t), w(t), u(t), K)=0, c \in \mathcal{C} \\
& \Delta\left(x\left(t_{s}^{+}\right), x\left(t_{s}^{-}\right)\right)=0 \\
& x_{\text {red }}(0)=\Pi x_{\text {red }}\left(T_{h}\right) \\
& h\left(x(t), u(t), w(t), f_{h}\right) \geq 0
\end{aligned}
$$

where $x=(\xi, \theta, \dot{\xi}, \dot{\theta})^{\top}$ indicates the state of the system.

We assume the mean speed in the locomotion direction is given, as we prescribe both the step length $S$ and the gait period $T$. In (7), we minimize the CoT. A cost index that realistically represents the energy consumption of an electrical powered geared motor is complex as this would require the modeling of both electrical and mechanical dynamics, see e.g. [28]. To enhance the tractability of the problem, we relate the actuator energy consumption to the squared torque which, with the squared power, is one of the two cost indices most commonly used in literature (e.g. [13]). This choice appears particularly appropriate due to the characteristics of SoftLegs actuators, that are electrically powered and backdrivable. This makes the energy efficiency of the system strictly related to the intensity of the torques. In (8), the function $F_{c}(\cdot)$ represents the dynamics of the system during the $c$-th contact phase. Because of periodicity and symmetry, the set of contact phases is $\mathcal{C}=\{1,2\}$, where 1 and 2 represent the single and the double support phase respectively. In (9), the function $\Delta(\cdot)$ represents the state discontinuity at the phase change occurring at time $t_{s} \in\left[0, T_{h}\right]$ where $T_{h}=T / 2$, caused by the impact of the floating foot with the ground. In (10) the gait is constrained to be periodic, by imposing the relation between $x_{\text {red }}(0)$ and $x_{\text {red }}\left(T_{h}\right)$. The additional inequality constraints, stated in (11), guarantee that the external contact wrench $w$ belongs to the static friction cone, that the contact is unilateral and that the Center of Pressure (CoP) is inside the support area. Moreover, limits on joint positions and velocities, as well as on motor torques, are imposed. Finally, during the single support phase, a minimum foot clearance $f_{h}$ is ensured between the floating foot and the ground. The OCP was solved numerically, by employing a direct collocation method [29]. Specifically, this method consists in: i) discretizing the time horizon into a finite sequence of time intervals, consistently parameterizing the state and control trajectories by finite sequences of optimization variables, ii) solving the resulting finite-dimensional nonlinear program (NLP) through a Newton-based solver.

Specifically, the problem was formulated in the CasADi framework [30] and solved numerically, using the interiorpoint solver IPOPT [31]. 485 OCPs have been solved successfully and, despite the problem complexity, each optimization lasted on average about 56 seconds. This method is not free 
from local minima and the global optimal solution search is out of the purpose of this work. Future works will include strategies to avoid local minima, such as convex relaxation of the nonlinear programming problem.

\section{REFERENCES}

[1] S. Collins, A. Ruina, R. Tedrake, and M. Wisse, "Efficient bipedal robots based on passive-dynamic walkers," Science, vol. 307, no. 5712, pp. $1082-1085,2005$

[2] A. Ramezani, J. W. Hurst, K. A. Hamed, and J. W. Grizzle, "Performance analysis and feedback control of atrias, a three-dimensional bipedal robot," Journal of Dynamic Systems, Measurement, and Control, vol. 136, no. 2, p. 021012, 2014.

[3] P. A. Bhounsule, J. Cortell, A. Grewal, B. Hendriksen, J. D. Karssen, C. Paul, and A. Ruina, "Low-bandwidth reflex-based control for lower power walking: $65 \mathrm{~km}$ on a single battery charge," The International Journal of Robotics Research, vol. 33, no. 10, pp. 1305-1321, 2014.

[4] The $w$ prize, for unprecedented speed, efficiency, and dexterity in terrestrail locomotion. http://www.wprize.org/aboutus.html.

[5] S. Kajita, F. Kanehiro, K. Kaneko, K. Fujiwara, K. Harada, K. Yokoi, and $\mathrm{H}$. Hirukawa, "Biped walking pattern generation by using preview control of zero-moment point," in Robotics and Automation, 2003. Proceedings. ICRA'03. IEEE International Conference on, vol. 2. IEEE, pp. $1620-1626$.

[6] M. A. Hopkins, D. W. Hong, and A. Leonessa, "Compliant locomotion using whole-body control and divergent component of motion tracking," in Robotics and Automation (ICRA), 2015 IEEE International Conference on. IEEE, 2015, pp. 5726-5733.

[7] R. T. Pierre-Brice Wieber and S. Kuindersma, "Modeling and control of legged robots," in Springer Handbook of Robotics, 2015.

[8] N. G. Tsagarakis, D. G. Caldwell, F. Negrello, W. Choi, L. Baccelliere, V. Loc, J. Noorden, L. Muratore, A. Margan, A. Cardellino, et al., "Walk-man: A high-performance humanoid platform for realistic environments," Journal of Field Robotics, vol. 34, no. 7, pp. 1225-1259, 2017.

[9] C. Knabe, R. Griffin, J. Burton, G. Cantor-Cooke, L. Dantanarayana, G. Day, O. Ebeling-Koning, E. Hahn, M. Hopkins, J. Neal, et al., "Team valor's escher: A novel electromechanical biped for the darpa robotics challenge," Journal of Field Robotics, 2017.

[10] F. Negrello, G. M., C. M. G., J. Malzahn, D. G. Caldwell, A. Bicchi, and N. G. Tsagarakis, "A modular compliant actuator for emerging high performance and fall-resilient humanoids," in HUMANOIDS2015, in 15th IEEE RAS Humanoids Conference, 2015.

[11] A. Velasco, G. M. Gasparri, M. Garabini, L. Malagia, P. Salaris, and A. Bicchi, "Soft-actuators in cyclic motion: Analytical optimization of stiffness and pre-load," in Humanoid Robots (Humanoids), 2013 13th IEEE-RAS International Conference on. IEEE, 2013, pp. 354-361.

[12] S. Haddadin, M. Weis, S. Wolf, and A. Albu-Schäffer, "Optimal control for maximizing link velocity of robotic variable stiffness joints," in IFAC world congress, 2011, pp. 6863-6871.

[13] A. Hereid, E. A. Cousineau, C. M. Hubicki, and A. D. Ames, "3d dynamic walking with underactuated humanoid robots: A direct collocation framework for optimizing hybrid zero dynamics," in Robotics and Automation (ICRA), 2016 IEEE International Conference on. IEEE, 2016, pp. 1447-1454.

[14] J. Kim, N. S. Pollard, and C. G. Atkeson, "Quadratic encoding of optimized humanoid walking," in Humanoid Robots (Humanoids), 2013 IEEE-RAS International Conference on. IEEE, pp. 300-306.

[15] X. Da, R. Hartley, and J. W. Grizzle, "Supervised learning for stabilizing underactuated bipedal robot locomotion, with outdoor experiments on the wave field," in Robotics and Automation (ICRA), 2017 IEEE International Conference on. IEEE, 2017, pp. 3476-3483.

[16] D. Clever, M. Harant, H. Koch, K. Mombaur, and D. Endres, "A novel approach for the generation of complex humanoid walking sequences based on a combination of optimal control and learning of movement primitives," Robotics and Autonomous Systems, vol. 83, pp. 287-298, 2016.

[17] S. Schaal, J. Peters, J. Nakanishi, and A. Ijspeert, "Learning movement primitives," Robotics Research, pp. 561-572, 2005.

[18] A. Safonova, J. K. Hodgins, and N. S. Pollard, "Synthesizing physically realistic human motion in low-dimensional, behavior-specific spaces," ACM Transactions on Graphics (TOG), vol. 23, no. 3, pp. 514-521, 2004.
[19] S. Levine, Y. Lee, V. Koltun, and Z. Popović, "Space-time planning with parameterized locomotion controllers," ACM Transactions on Graphics (TOG), vol. 30, no. 3, p. 23, 2011.

[20] C. Della Santina, M. Bianchi, G. Grioli, F. Angelini, M. G. Catalano, M. Garabini, and A. Bicchi, "Feedback and feedforward control for soft robots," IEEE Robotics \& Automation Magazine.

[21] G. Pratt, M. M. Williamson, et al., "Series elastic actuators," in Intelligent Robots and Systems 95.'Human Robot Interaction and Cooperative Robots', Proceedings. 1995 IEEE/RSJ International Conference on, vol. 1. IEEE, 1995, pp. 399-406.

[22] A. D. Kuo, "A simple model of bipedal walking predicts the preferred speed-step length relationship," Journal of biomechanical engineering, vol. 123 , no. 3, pp. 264-269, 2001

[23] N. Smit-Anseeuw, R. Gleason, R. Vasudevan, and C. D. Remy, "The energetic benefit of robotic gait selectiona case study on the robot ramone," IEEE Robotics and Automation Letters, vol. 2, no. 2, pp. 1124 1131, 2017.

[24] Catalano et al., "Vsa-cubebot: A modular variable stiffness platform for multiple degrees of freedom robots," in Robotics and Automation (ICRA), IEEE International Conference on. IEEE, 2011, pp. 50905095.

[25] Optoforce sensors. [Online]. Available: http://optoforce.com/

[26] C. Della Santina et al., "The quest for natural machine motion: An open platform to fast-prototyping articulated soft robots," IEEE Robotics \& Automation Magazine, 2017.

[27] T. Marcucci, M. Garabini, G. M. Gasparri, A. Artoni, M. Gabiccini, and A. Bicchi, "Parametric trajectory libraries for online motion planning with application to softrobots," in International Symposium on Robotics Research (ISRR), 2017 IEEE International Conference on.

[28] T. Verstraten, P. Beckerle, R. Furnémont, G. Mathijssen, B. Vanderborght, and D. Lefeber, "Series and parallel elastic actuation: Impact of natural dynamics on power and energy consumption," Mechanism and Machine Theory, vol. 102, pp. 232-246, 2016.

[29] C. R. Hargraves and S. W. Paris, "Direct trajectory optimization using nonlinear programming and collocation," Journal of Guidance, Control, and Dynamics, vol. 10, no. 4, pp. 338-342, 1987.

[30] J. Andersson, J. Åkesson, and M. Diehl, "Casadi: A symbolic package for automatic differentiation and optimal control," in Recent Advances in Algorithmic Differentiation. Springer, 2012, pp. 297-307.

[31] A. Wächter and L. T. Biegler, "On the implementation of an interiorpoint filter line-search algorithm for large-scale nonlinear programming," Mathematical Programming, vol. 106, no. 1, pp. 25-57, 2006. 